\title{
Biocatalytic induction of supramolecular order
}

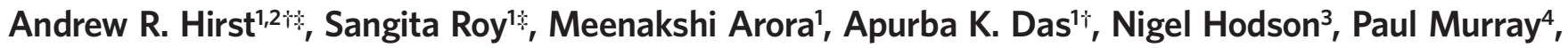 \\ Stephen Marshall ${ }^{4}$, Nadeem Javid ${ }^{5}$, Jan Sefcik ${ }^{5}$, Job Boekhoven ${ }^{6}$, Jan H. van Esch ${ }^{6}$, \\ Stefano Santabarbara ${ }^{7 \dagger}$, Neil T. Hunt ${ }^{7}$ and Rein V. Ulijn ${ }^{1 \star}$
}

\begin{abstract}
Supramolecular gels, which demonstrate tunable functionalities, have attracted much interest in a range of areas, including healthcare, environmental protection and energy-related technologies. Preparing these materials in a reliable manner is challenging, with an increased level of kinetic defects observed at higher self-assembly rates. Here, by combining biocatalysis and molecular self-assembly, we have shown the ability to more quickly access higher-ordered structures. By simply increasing enzyme concentration, supramolecular order expressed at molecular, nano- and micro-levels is dramatically enhanced, and, importantly, the gelator concentrations remain identical. Amphiphile molecules were prepared by attaching an aromatic moiety to a dipeptide backbone capped with a methyl ester. Their self-assembly was induced by an enzyme that hydrolysed the ester. Different enzyme concentrations altered the catalytic activity and size of the enzyme clusters, affecting their mobility. This allowed structurally diverse materials that represent local minima in the free energy landscape to be accessed based on a single gelator structure.
\end{abstract}

M olecular self-assembly ${ }^{1-7}$ can be controlled using a variety of stimuli, including chemical ${ }^{8,9}$ and mechanical ${ }^{10}$ triggers, as well as X-rays ${ }^{11}$. Although the traditional premise in selfassembly suggests that supramolecular material properties can be fully encoded into molecular building blocks, it is increasingly apparent that the chosen self-assembly pathway is central to the final structure and its material functionality. Biocatalytic control of self-assembly systems is a novel direction for laboratory-based self-assembly ${ }^{12-17}$, although it is omnipresent in the biological world. Indeed, enzymatically controlled self-assembly and disassembly underlies vital processes such as cell movement, intracellular transport and muscle contraction. In chemists' hands, the combination of biocatalysis and molecular self-assembly has recently emerged as a powerful new approach to make novel stimuli-responsive molecular materials ${ }^{12-17}$. We believe that catalytic control of self-assembly provides important new methodology beyond such triggering of material transitions. In particular, the combination of biological selectivity, localized action and operation under constant, physiological conditions provides a new methodology for bottomup nanofabrication of future soft materials and devices, allowing for unprecedented control of supramolecular order.

Here, we focus on the control of supramolecular order with few defects. In principle, there are two possible approaches to defect reduction-either improving the fidelity of the self-assembly process (avoiding defects) or using fully reversible systems that operate under thermodynamic control (repairing defects). The latter approach is generally slow and only applicable to cases where the desired structure represents the global equilibrium state and where the system is fully reversible, that is, under thermodynamic control ${ }^{16}$. Many structures of interest, however, represent local thermodynamic minima ${ }^{18,19}$. Such structures can potentially be locked by appropriately tuning the assembling condition $s^{20}$.
We hypothesized that by taking advantage of the unique localized nucleation and growth mechanism observed for biocatalysed systems, in combination with kinetic control, it would be possible to direct the self-assembling process towards such local thermodynamic minima. Here, using the enzyme subtilisin as a biocatalyst, we demonstrate the formation of non-equilibrium supramolecular structures that self-assemble more quickly, and are more highly ordered as enzyme concentration increases.

\section{Results and discussion}

Biocatalytic self-assembly and gelation. Self-assembling peptides are versatile building blocks for the production of nanostructures ${ }^{21-23}$. Our system focuses on a number of related precursors based on aromatic peptide amphiphiles ${ }^{16,24,25}$, namely Fmoc-dipeptide methyl esters, where Fmoc is the chromophore $\mathrm{N}$-(fluorenyl-9methoxycarbonyl), and the dipeptides are FF (1), FY (2), YL (3), VL (4) and FL (5), where F is the amino acid phenylalanine, $\mathrm{Y}$ is tyrosine, $\mathrm{L}$ is leucine and $\mathrm{V}$ is valine (see Fig. 1a, Table 1 and Supplementary Table S1). Molecular self-assembly is initiated by subtilisin-a hydrolytic enzyme from Bacillus licheniformiswhich hydrolyses the methyl ester to form a peptide derivative that self-assembles (Fig. 1a). In these systems, nucleation and early-stage structure growth are spatially confined at the site of enzyme action, as shown diagrammatically in Fig. $1 b^{16}$. Hence, it appears sensible to expect that the properties of the self-assembling supramolecular structures could be affected by these catalytic centres. Figure 1c shows typical nucleation and initiation of fibre growth as captured by atomic force microscopy (AFM). In each case, after $1 \mathrm{~h}$ of incubation at $55^{\circ} \mathrm{C}$ and $\mathrm{pH} 8$, followed by cooling to room temperature, self-supporting gels were formed (Fig. 1d). Fourier transform infrared (FTIR) analysis (Fig. 1e) revealed the formation of anti-parallel $\beta$-sheet structures upon cooling to room temperature.

${ }^{1}$ WestCHEM, Department of Pure \& Applied Chemistry, University of Strathclyde, Glasgow, Scotland, UK, ${ }^{2}$ Manchester Interdisciplinary Biocentre, University of Manchester, UK, ${ }^{3}$ BioAFM Facility, Faculty of Medical and Human Sciences, The University of Manchester, Manchester, UK, ${ }^{4}$ Department of EEE, University of Strathclyde, Glasgow, Scotland, UK, ${ }^{5}$ Department of Chemical and Process Engineering, University of Strathclyde, Glasgow, Scotland, UK, ${ }^{6}$ Delft University of Technology, Delft, Netherlands, ${ }^{7}$ Department of Physics, University of Strathclyde, SUPA, Glasgow, Scotland, UK; 'Present address: School of Physics and Astronomy, E C Stoner Building, University of Leeds, Leeds, LS2 9JT, UK (A.R.H.); Department of Chemistry, Indian Institute of Technology Indore, IET, DAVV Campus, Khandwa Road, Indore 452017, India (A.K.D.); Istituto di Biofisica, Consiglio Nazionale delle Ricerche, Via Celoria 26, 20133 Milano, Italy (S.S); *These authors contributed equally to this work. *e-mail: rein.ulijn@strath.ac.uk 
a
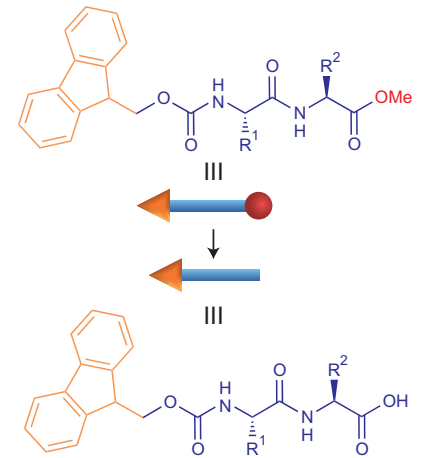

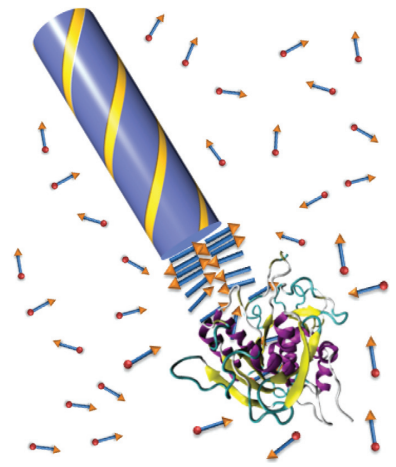

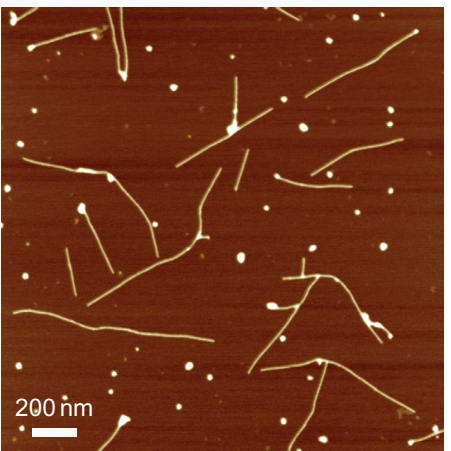
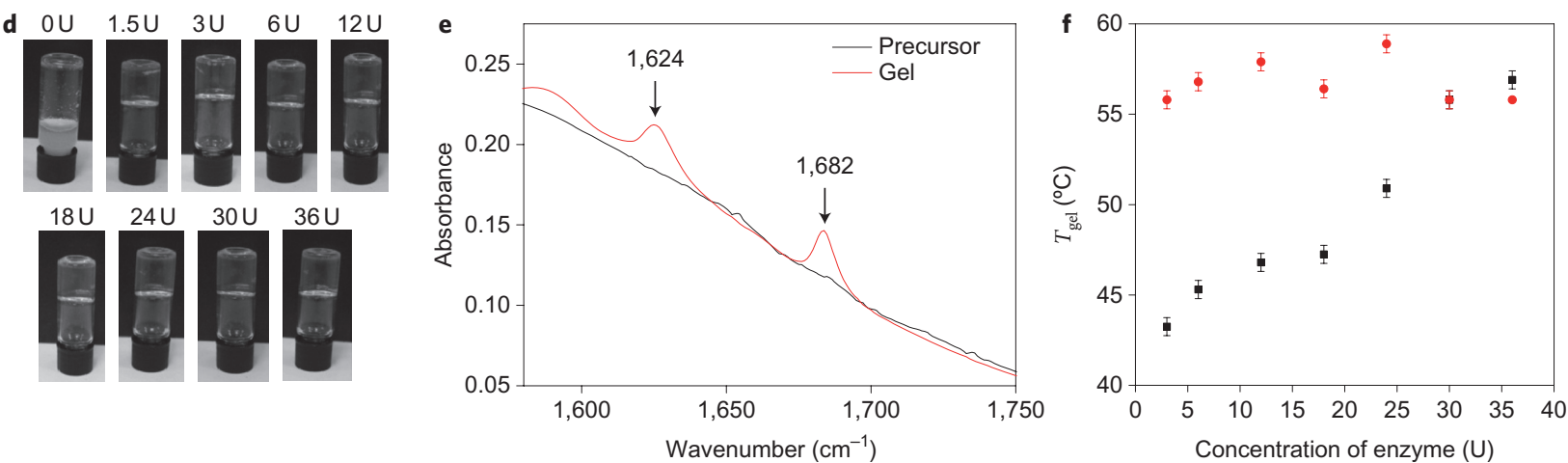

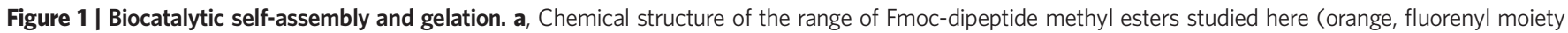
of the Fmoc group; blue, dipeptide sequence; red, enzyme cleavable methoxy group). Subtilisin catalyses hydrolysis of these esters to Fmoc-peptide gelators. b, Schematic of nucleation and growth mechanism of self-assembly controlled by subtilisin. $\mathbf{c}$, AFM analysis of initial stages of the self-assembly process ( $12 \mathrm{U}, 20 \mathrm{~min}$ at $55^{\circ} \mathrm{C}$, followed by cooling to room temperature and air drying. Here, $1 \mathrm{U}$ is equivalent to the amount of enzyme that hydrolyses casein to produce a colour equivalent to $1 \mathrm{mmol}$ of tyrosine per minute at $\mathrm{pH} 7.5$ at $37^{\circ} \mathrm{C}$ ), showing the formation of clusters and fibres. d, Self-supporting, optically transparent gels are formed in each case. e, FTIR spectrum of gel-phase materials showing formation of the antiparallel $\beta$-sheet conformation. $\mathbf{f}$, Melting temperature $\left(T_{\text {gel }}\right)$ of gels formed catalytically (black) and by a heating-cooling cycle (red) at different enzyme concentrations. The error bars show a standard error corresponding to $\pm 0.5^{\circ} \mathrm{C}$.

Table 1 | Structures of the amino acids used for the dipeptides.

Tyrosine (Y)
Peucine (L)
Valine (V)

Further analysis by circular dichroism (CD) and fluorescence (vide infra) was consistent with previous observations reported for a series of closely related aromatic peptide amphiphiles ${ }^{16,24,25}$. In these cases, nano-sized fibres ( $\sim 10 \mathrm{~nm}$ in diameter) were composed of several $\beta$ sheets that were locked together by means of $\pi$-stacking interactions. We will first discuss the final supramolecular structures that were obtained at the different enzyme concentrations. The processes that took place during the enzymatic conversion and cooling stages will then be discussed.
The rate of catalytic self-assembly could be tuned simply by changing the amount of enzyme present in the system. A range of enzyme concentrations, between 1.5 and $36 \mathrm{U}$ (units), were therefore selected but with otherwise identical treatment $\left(1 \mathrm{~h}\right.$ at $55^{\circ} \mathrm{C}$, followed by cooling to room temperature, then left for $72 \mathrm{~h}$ to ensure that structures were kinetically locked). During this time, enzymatic conversion was dictated by enzyme concentration, with conversions of between 55 and $99 \%$ after 20 min, reaching $>96 \%$ for all enzyme concentrations in each case, with complete conversions (>99\%) observed after cooling (Supplementary Fig. S1). The resulting materials were structurally different, as was immediately evident from a comparison of the melting behaviour of these gels, which showed that higher enzyme concentrations gave rise to increasingly stable gels with melting temperatures ranging from $T_{\text {gel }}=42{ }^{\circ} \mathrm{C}$ to $58^{\circ} \mathrm{C}$. These $T_{\text {gel }}$ values relate to a hard gel boundary point, that is, the temperature at which solvent is released from the material, providing an indication of the network morphology. When they were heated to $90{ }^{\circ} \mathrm{C}$ to disrupt the molecular order and then cooled, the same $T_{\text {gel }}$ values were observed in each case ( $58{ }^{\circ} \mathrm{C}$; Fig. 1f). The differences in the properties of the enzymatically produced gels therefore result from their supramolecular organization. This observation provides strong evidence that kinetically trapped structures are formed under catalytic control, but the imposition of a subsequent heating/cooling cycle gives the thermodynamically favoured state. In a previous study by Pochan and colleagues, different moduli were observed at faster assembly rates for salt-induced self-assembly and were related to molecular-level defects that had resulted in changes in the network properties ${ }^{26}$. 


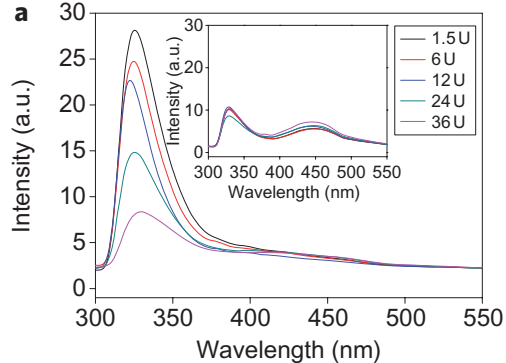

d

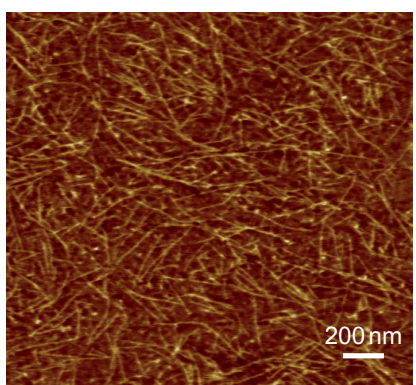

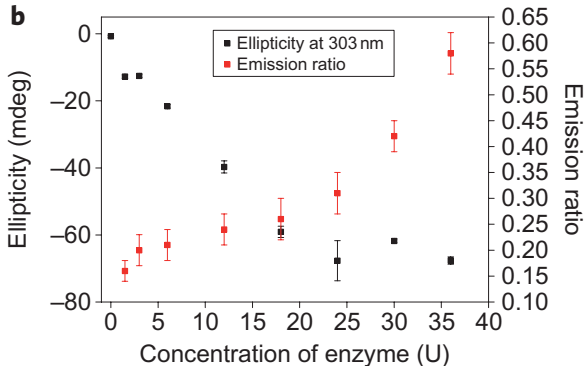

e

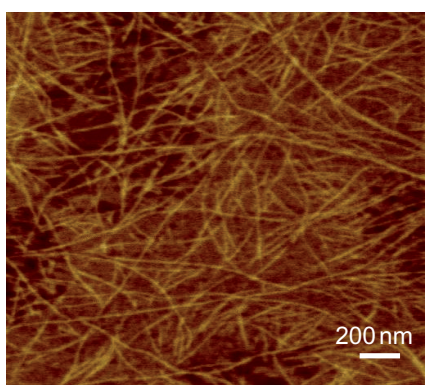

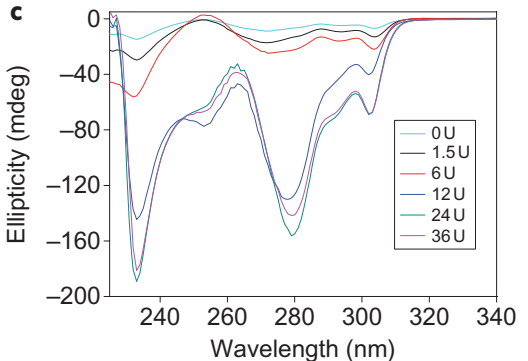

$\mathbf{f}$

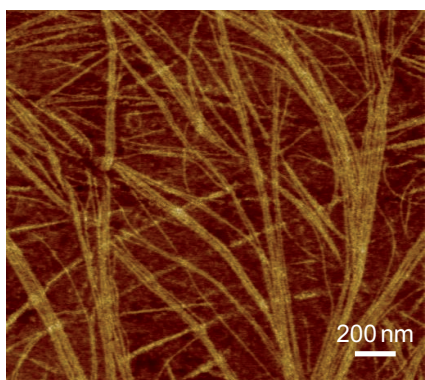

Figure 2 | Spectroscopic and structural evidence of catalytically induced supramolecular order. a, Emission spectra (excitation at $280 \mathrm{~nm}$ ) of Fmoc-YL gel obtained at different enzyme concentrations. Inset: Emission spectra of the gels after a heating-cooling cycle. $\mathbf{b}$, Correlation of the increase in ellipticity at $303 \mathrm{~nm}$ with emission ratio. For ellipticity and emission ratio measurement, experimental data was acquired in triplicate. The average ellipticity at $303 \mathrm{~nm}$ was plotted and the error bars show the standard deviation of the three measurements, corresponding to values in the range $\pm(-0.3$ to -1.07$)$. For emission ratio, the average of the three experiments was plotted and error bars correspond to values in the range $\pm 0.02-0.04$. c, CD spectra of supramolecular hydrogel of Fmoc-YL, showing that higher-order chirality is induced with increasing enzyme concentrations. d-f, AFM images of the gels at 1.5, 12 and $30 \cup$ (after $72 \mathrm{~h}$ ).

Spectroscopic evidence of catalytically induced order. To acquire more insights at the supramolecular level, the gels were characterized by spectroscopic methods. The principal molecular interactions driving the self-assembly of aromatic peptide amphiphiles are weak hydrophobic forces, leading to $\pi-\pi$ stacking between the fluorenyl chromophores, and hydrogen bonding between the dipeptides. Fluorescence emission spectra (shown in Fig. 2a) principally monitored the emission of the fluorenyl moieties, and in this case displayed two characteristic peaks, a narrow band centred at $320 \mathrm{~nm}$ and relatively broad, redshifted structure displaying maxima between 420 and $440 \mathrm{~nm}$. The former is assigned to emission of Fmoc-YL monomers, the latter to the emission from the low-energy exciton state(s) assigned to excimer(s) promoted by $\pi$-stacking of Fmoc moieties. In addition to hydrogen bonding, $\pi-\pi$ stacking between fluorenyl moieties is an important feature that underpins fibrillar molecular self-assembly, and it is observed in gels produced at every concentration of subtilisin. Nevertheless, it is clear that the relative intensity of the monomeric $(320 \mathrm{~nm})$ and excimeric $(420 \mathrm{~nm})$ emission bands depends on the amount of catalyst present in the reaction mixture. In particular, the monomeric emission is progressively quenched with respect to excimer emission by increasing the quantities of enzyme (Fig. 2b) up to $36 \mathrm{U}$. No further increase was observed at higher catalyst concentrations. The analysis of fluorescence emission spectra provides a clear indication that enhanced catalyst concentrations promote the formation of extended $\pi-\pi$ interactions among the fluorenyl moieties. This leads to the formation of extended onedimensional arrays that can be interpreted as a more ordered supramolecular structure. Upon heating of the locked gels above their $T_{\text {gel }}$, followed by cooling to room temperature, the same spectra were observed in each case (Fig. 2a, inset), again providing clear evidence of catalytically induced access of local thermodynamic minima that can be thermally unlocked.

The occurrence of extensive excitonic interaction amongst the Fmoc chromophores, as indicated by fluorescence emission measurements, is expected to give rise to pronounced CD signals. Figure $2 \mathrm{c}$ shows that not only is this the case at all concentrations of subtilisin, but the magnitude of $\mathrm{CD}$ is directly correlated with quenching of monomeric Fmoc emission at $320 \mathrm{~nm}$ (Fig. 2b). It should be noted that no significant linear dichroism contribution was observed (Supplementary Fig. S2). The amide regions of the spectra $(200-230 \mathrm{~nm})$ were noisy due to high absorbance in this region, with contributions from the enzyme and the peptide gelators (Supplementary Fig. S2). However, we observed peaks at 280, 294 and $303 \mathrm{~nm}$, which coincide with Fmoc absorption. CD spectra are typically recorded in large macromolecules, such as protein, DNA and even membrane ultrastructures, where the size of the supramolecular structure is close to, or exceeds that, of the measuring wavelength ${ }^{26}$. This situation also occurs in the self-assembling structures described here (see AFM data, Fig. 2d-f). In such systems, CD signals result from chiral organization of the supramolecular assembly, rather than from inherent molecular chirality within the chromophore itself. From the results of Fig. 2c, two main conclusions can be drawn. First, the sign of the CD is the same for all enzyme concentrations tested, indicating a bias in the self-assembled structure for a single-handed structure. Second, and more importantly, the level of chiral organization in the selfassembled state can be controlled by the amount of enzyme. This suggests that increasing the amount of enzyme facilitates the transcription of chiral information from the molecular-scale building blocks to the self-assembled state, and in the presence of relatively high enzyme amounts the chirality of the system can be remarkably enhanced. After melting and cooling of the gels, similar CD spectra were observed for each sample (Supplementary Fig. S2c), but with significant differences in the spectral features when compared with the samples before melting.

Formation of catalytic clusters. Analysis by AFM revealed (Figs 2d-f,3a and Supplementary Fig. S3) that the nanometre- to micrometre-scale self-assembled network structure was directly controlled by the amount of enzyme, with lower enzyme concentrations giving rise to 

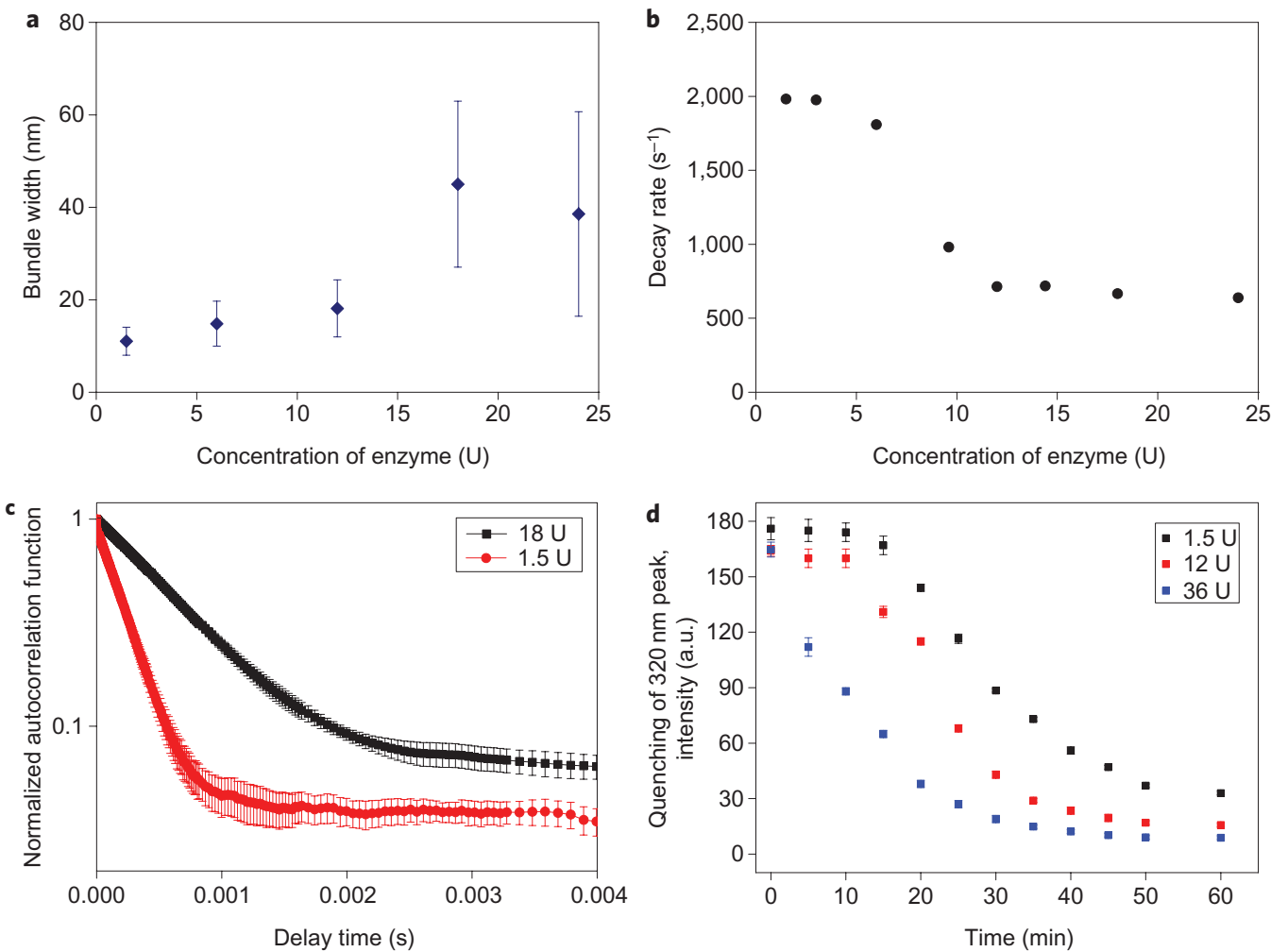

Figure $\mathbf{3}$ | Evidence of catalytic clusters and cooperativity. a, Image analysis revealing the extent of bundling in relation to enzyme concentration. Error bars show the standard deviation, corresponding to a value in the range $\pm 3-22 \mathrm{~nm}$. $\mathbf{b}$, DLS results show a slowing down of density fluctuation decay at higher enzyme concentrations. The 30 and $36 \mathrm{U}$ samples resulted in precipitates and were therefore excluded. c, Autocorrelation function showing a slower relaxation mode for higher enzyme concentrations. The experiments were performed six times and the resulting autocorrelation functions were averaged; standard deviations are shown. d, Quenching of intensity of the $320 \mathrm{~nm}$ peak for low (1.25 U), medium (12 U) and high (36 U) enzyme concentrations during heating at $55^{\circ} \mathrm{C}$ for $1 \mathrm{~h}$. Experimental data for quenching was acquired in triplicate and the average intensity values of the three measurements were plotted and error bars correspond to standard deviation in the range $\pm 1-6 \mathrm{AU}$.

shorter fibres that were less bundled (Fig. 2d). Quantitatively, this is supported by advanced digital image-processing techniques, which were used to generate a graph showing the width distribution of the fibre bundles (Fig. 3a). We propose that a combination of two factors dictates the remarkable catalytic induced long-range stacking described here: enzymes acting cooperatively within catalytic clusters and the restricted mobility of these clusters as enzyme concentrations increase. Dynamic light-scattering (DLS) analysis of enzyme solutions at $55^{\circ} \mathrm{C}$ demonstrated that the initial decay rate of density fluctuations decreased (Fig. $3 \mathrm{~b}$ ) and slower relaxation modes became more prominent (Fig. 3c) with increasing enzyme concentrations. Samples with enzyme concentrations of 30 and $36 \mathrm{U}$ resulted in precipitates and were therefore excluded from further analysis. DLS results showed that enzymes formed extended clusters at all concentrations tested, because decay rates that are an order of magnitude higher would be expected for relaxation due to Brownian motion of individual enzyme molecules. Assuming that the initial decay rates are due to unhindered Brownian motion of clusters, their mean hydrodynamic radius would vary from 85 to $260 \mathrm{~nm}$ with increasing enzyme concentration. However, these clusters are not completely free to move around, as indicated by the presence of slow relaxation modes.

Slow relaxation modes in the DLS autocorrelation function (Fig. 3c) were accompanied by considerable slow-down of the initial decay rates at higher enzyme concentrations (Fig. 3b). These observations indicate that extended interconnected networks are formed in these samples, with networks becoming more rigid with an increasing degree of interconnection, similar to previous observations in colloidal, protein and polymer solutions ${ }^{27-29}$. It is likely that the mobility of enzyme clusters within these increasingly rigid interconnected networks is reduced, reducing discontinuities in fibre assembly and thereby favouring the formation of longer fibres, as observed by AFM. Indeed, there is a clear inverse correlation between the bundle widths observed and decay rates (Fig. 3a,b).

Dynamics: higher supramolecular order faster. To gain further insight into the processes that underpin structure formation, spectral changes were followed during enzymatic reaction using fluorescence. It was found that, for all enzyme concentrations, the supramolecular organization of fluorenyl groups (as indicated by quenching of the $320 \mathrm{~nm}$ band) occurred during enzymatic conversion at $55{ }^{\circ} \mathrm{C}$. Figure $3 \mathrm{~d}$ shows spectral changes between low (1.5 U), medium (12 U) and high (36 U) enzyme concentrations, clearly showing more rapid formation of quenched structures for the high concentration. These values showed only minor changes after cooling and for up to $72 \mathrm{~h}$. In contrast, there was no detectable $\beta$-sheet signal apparent from the FTIR results during the enzymatic conversion (Supplementary Fig. S4); however, clear peaks emerged upon cooling, with similar spectra appearing for all enzyme concentrations tested. Clearly, molecular order driven by $\pi$-stacking interactions is induced before gelation, and it is locked into a $\beta$-sheet structure upon cooling.

For the Fmoc-YL-OMe (3) system described above, some gels (24-36 U) were formed at or above their $T_{\text {gel }}$, whereas others (1-20 U) were formed below $T_{\text {gel }}$. Although gelation is not observed before cooling, one might expect the $T_{\text {gel }}$ compared to the reaction temperature to have an impact on the gel properties. To study this effect, two additional gelators were investigated with different $T_{\text {gel }}$ 
values $\left(\sim 47{ }^{\circ} \mathrm{C}\right.$, Fmoc-FY; $42{ }^{\circ} \mathrm{C}$, Fmoc-VL). These were studied at enzyme concentrations of 1.5, 6, 12, 24 and $36 \mathrm{U}$. Fmoc-FY showed evidence of catalytic induced quenching of the monomeric peak, combined with an enhanced excimer peak, as well as an enzymatically induced $T_{\text {gel }}$ that varied in value between $\sim 34$ and $43{ }^{\circ} \mathrm{C}$ (Supplementary Fig. S5a-c). A more modest catalytically induced quenching of fluorescence and absence of induced $T_{\text {gel }}$ was observed for the lower melting Fmoc-VL, which is likely to be less structured at the assembly temperature of $55{ }^{\circ} \mathrm{C}$ (Supplementary Fig. S5d-f). These results suggest that catalytic locking of order is most pronounced under conditions where self-assembly, but not necessarily gel formation, is favoured.

Because of the correlation between the intensity of the CD signal, the quenching of monomeric Fmoc emission (Fig. 2), the observed gel melting temperatures (Fig. 1) and enhanced network formation (Fig. 2), it is apparent that the bundled chiral supramolecular structures represent a highly ordered state of these systems that is preferentially obtained at higher catalyst concentrations. It is worth noting that the formation of well-aligned extended one-dimensional $\pi$-stacked nanofibres is of relevance to supramolecular electronics ${ }^{30-33}$. The results shown here imply that supramolecular electro-conductivity may be induced and controlled by catalytic self-assembly.

In summary, we have demonstrated that by modulating the amount of catalyst we can access a library of structurally diverse molecular and network assemblies in which, importantly, all gelator concentrations are identical. The ability to kinetically induce molecular order in a controlled and reproducible manner allows a high level of control to be exerted over the assembly process, thereby enabling access to structurally diverse self-assembled materials that are inaccessible via conventional self-assembly. We provide evidence of a mechanism in which the catalytic activity and mobility of biocatalytic clusters dictate the induced supramolecular order observed. These results suggest an important role for engineered catalytic particles in the molecular self-assembly of next-generation soft nanomaterials and devices.

\section{Methods}

Catalytic self assembly. Fmoc-YL-OMe $\left(10 \mathrm{mmol} \mathrm{kg}^{-1}\right)$ was dispersed in a $1 \mathrm{ml}$ volume of $100 \mathrm{mM}$ sodium phosphate buffer $(\mathrm{pH} 8)$ in the presence of varying concentrations of subtilisin (1.25-60.0 $\mu$ l (Sigma Aldrich, catalogue number P4860; LOT 056K1213). The mixture was vortexed (30 s) and sonicated on ice for $20 \mathrm{~min}$ to ensure that a homogeneous mixture was obtained, and the low temperature ensures that no enzymatic conversion occurs up to this point. This was followed by heating either in an oil bath or within the spectrophotometer using a temperature-controlled cuvet, at $55{ }^{\circ} \mathrm{C}$ for $60 \mathrm{~min}$ to allow enzymatic conversion to occur. The selfassembling system was then allowed to cool to room temperature. The gel samples were then left to stand for periods of $72 \mathrm{~h}$ before experimental measurements were performed. Gelation was considered to have occurred when a homogeneous 'solid-like' material was obtained that exhibited no gravitational flow. The thermally reversible gel-sol transition temperature $\left(T_{\text {gel }}\right)$ was determined using a ball dropping methodology.

High-performance liquid chromatography. A Dionex P680 high-performance liquid chromatography pump was used to quantify conversions of the enzymatic reaction. A $50 \mu \mathrm{l}$ sample was injected onto a Macherey-Nagel $\mathrm{C}_{18}$ column with a length of $250 \mathrm{~mm}$ and an internal diameter of $4.6 \mathrm{~mm}$ and 5-mm fused silica particles at a flow rate of $1 \mathrm{ml} \mathrm{min}{ }^{-1}$. The eluting solvent system had a linear gradient of $20 \%(\mathrm{v} / \mathrm{v})$ acetonitrile in water for $4 \mathrm{~min}$, gradually rising to $80 \%(\mathrm{v} / \mathrm{v})$ acetonitrile in water at $35 \mathrm{~min}$. This concentration was kept constant until $40 \mathrm{~min}$ when the gradient was decreased to $20 \%(\mathrm{v} / \mathrm{v})$ acetonitrile in water at $42 \mathrm{~min}$. Sample preparation involved mixing $50 \mu \mathrm{l}$ of gel with acetonitrile-water $(950 \mu \mathrm{l}$, 50:50 mixture) containing $0.1 \%$ trifluoroacetic acid. The purity of each identified peak was determined by UV detection at $280 \mathrm{~nm}$.

Circular dichroism (CD). Spectra were measured on a Jasco J815 spectropolarimeter with 1-s integrations, a step size of $1 \mathrm{~nm}$ and a single acquisition with a slit width of $1 \mathrm{~nm}$ due to the dynamic nature of the system. Experimental data were acquired in triplicate.

Fluorescence spectroscopy. Fluorescence emission spectra were measured on a Jasco FP-6500 spectrofluorometer with light measured orthogonally to the excitation light, with excitation at $280 \mathrm{~nm}$ and an emission data range between 300 and $600 \mathrm{~nm}$.
Atomic force microscopy (AFM). Glass cover slips (Agar Scientific) were cleaned overnight in ethanol (Aldrich), air-dried and adhered to AFM support stubs (Agar Scientific) using clear nail varnish. Gel samples were prepared as previously described, deposited on the glass surface, and allowed to air dry overnight. Samples were imaged by intermittent contact mode in air using a Veeco Multimode atomic force microscope with a Nanoscope IIIa controller and an 'E' scanner. Imaging was performed using silicon probes (OTESPA, Veeco Instruments S.A.S.) with a nominal spring constant of $42 \mathrm{~N} \mathrm{~m}^{-1}$. Cantilever oscillation varied between 300 and $350 \mathrm{kHz}$ and the drive amplitude was determined by the Nanoscope software. The setpoint was adjusted to just below the point at which tip - sample interaction was lost. Height and phase images with scan sizes of either 5 or $2 \mu \mathrm{m}^{2}$ were captured at a scan rate of $1.49 \mathrm{~Hz}$ and at a relative humidity of $<40 \%$. Data were first-order flattened using the Nanoscope software before image export. The instrument was periodically calibrated using a grating with $180-\mathrm{nm}$-deep, $10-\mu \mathrm{m}^{2}$ depressions.

FTIR spectroscopy. FTIR spectra were acquired in a Bruker Vertex spectrometer with a spectral resolution of $2 \mathrm{~cm}^{-1}$. The spectra were obtained by averaging 64 interferograms for each sample. Measurements were performed in a standard IR cuvette (Harrick Scientific), in which the sample was contained between two $\mathrm{CaF}_{2}$ windows (thickness, $2 \mathrm{~mm}$ ) separated by a $25-\mu \mathrm{m}$ PTFE spacer. All sample manipulations were performed in a glove box to minimize interference from carbon dioxide and water vapour; $\mathrm{D}_{2} \mathrm{O}$ was used as the solvent for all the IR measurements.

Dynamic light scattering (DLS). The required amounts of protease solution were mixed with $100 \mathrm{mM}$ phosphate buffer filtered with a $0.25 \mu \mathrm{m}$ Anotop filter to achieve the required protein concentrations of $1.5,3,6,12,18,24,30$ and $36 \mathrm{U} \mathrm{ml}^{-1}$. The samples were vortexed for $1 \mathrm{~min}$ and transferred to borosilicate glass cuvettes for scattering measurements. DLS experiments were carried out using a 3D LS spectrometer (LS instruments) using vertically polarized He-Ne laser light $(25 \mathrm{~mW}$, with a wavelength of $632.8 \mathrm{~nm}$ ) with an avalanche photodiode detector at a scattering angle of $90^{\circ}$. The samples were heated to achieve a temperature of $55^{\circ} \mathrm{C}$ using a circulating water bath. The intensity autocorrelation function $g_{2}(\tau)-1$ was recorded from time zero up to $25 \mathrm{~min}$ to determine the evolution of the average apparent hydrodynamic radius with time. For all measurements, the decay of $g_{2}(\tau)-1$ was followed up to large values of delay time $\tau$ to reach the baseline. The autocorrelation functions were analysed by means of the cumulant method to determine the average apparent diffusion coefficient $D$. The decay of the autocorrelation function was modelled as $g_{1}(\tau)=\left(g_{2}(\tau)-1\right)^{1 / 2}=\exp (-\Gamma t)$, where $\Gamma=D q^{2}$ is the decay rate, $q=(4 \pi n / \lambda) \sin (\theta / 2)$ is the scattering vector magnitude, $n$ is the refractive index of the solvent, $\theta$ is the scattering angle and $\lambda$ is the wavelength of the laser. The average hydrodynamic radius $r_{\mathrm{H}}$ can be calculated from the StokesEinstein equation, $r_{\mathrm{H}}=k_{\mathrm{B}} T / 6 \pi \eta \mathrm{D}$, where $k_{\mathrm{B}}$ is the Boltzmann constant, $T$ is the absolute temperature and $\eta$ is the solvent viscosity at the given temperature.

\section{Received 6 April 2010; accepted 23 August 2010;} published online 10 October 2010

\section{References}

1. Estroff, L. A. \& Hamilton, A. D. Water gelation by small organic molecules. Chem. Rev. 104, 1201-1217 (2004).

2. Lehn, J. M. Supramolecular Chemistry-Concepts and Perspectives (VCH Weinheim, 1995).

3. Whitesides, G. M. \& Boncheva, M. Beyond molecules: self-assembly of mesoscopic and macroscopic components. Proc. Natl Acad. Sci. USA 99, 4769-4774 (2002)

4. Hirst, A. R. et al. High-tech applications of self-assembling supramolecular nanostructured gel-phase materials: from regenerative medicine to electronic devices. Angew. Chem. Int. Ed. 47, 8002-8018 (2008).

5. Capito, R. M., Azevedo, H. S., Velichko, Y. S., Mata, A. \& Stupp, S. I. Self-assembly of large and small molecules into hierarchically ordered sacs and membranes. Science 319, 1812-1816 (2008).

6. Wang, Q. et al. High-water-content mouldable hydrogels by mixing clay and a dendritic molecular binder. Nature 463, 339-343 (2010).

7. Kiyonaka, S. et al. Semi-wet peptide/protein array using supramolecular hydrogel. Nature Mater. 3, 58-64 (2004).

8. Jonkheijm, P., van der Schoot, P., Schenning, A. P. H. J. \& Meijer, E. W. Probing the solvent-assisted nucleation pathway in chemical self-assembly. Science 313, 80-83 (2006).

9. Lloyd, G. O. \& Steed, J. W. Anion-tuning of supramolecular gel properties. Nature Chem. 1, 437-442 (2009).

10. Carnall, J. M. A. et al. Mechanosensitive self-replication driven by selforganization. Science 327, 1502-1506 (2010)

11. Cui, H. et al. Spontaneous and X-ray triggered crystallization at long range in self-assembling filament networks. Science 327, 555-559 (2010).

12. Winkler, S., Wilson, D. \& Kaplan, D. L. Controlling $\beta$-sheet assembly in genetically engineered silk by enzymatic phosphorylation/dephosphorylation. Biochemistry 39, 12739-12746 (2000). 
13. Hu, B. H. \& Messersmith, P. B. Rational design of transglutaminase substrate peptides for rapid enzymatic formation of hydrogels. J. Am. Chem. Soc. 125, 14298-14299 (2003).

14. Yang, Z. M. et al. Enzymatic formation of supramolecular hydrogels. Adv. Mater. 16, 1440-1444 (2004).

15. Um, S. H. et al. Enzyme-catalysed assembly of DNA hydrogel. Nature Mater. 5, 797-801 (2006).

16. Williams, R. J. et al. Enzyme-assisted self-assembly under thermodynamic control. Nature Nanotech. 4, 19-24 (2009).

17. Adler-Abramovich, L., Perry, R., Sagi, A., Gazit, E. \& Shabat D. Controlled assembly of peptide nanotubes triggered by enzymatic activation of self-immolative dendrimers. ChemBioChem 8, 859-862 (2007).

18. Cui, H., Chen, Z., Zhong, S., Wooley, K. L. \& Pochan, D. J. Block copolymer assembly via kinetic control. Science 317, 647-650 (2007).

19. Yamamoto, T. et al. Stabilization of a kinetically favored nanostructure: surface ROMP of self-assembled conductive nanocoils from a norbornene-appended hexa-peri-hexabenzocoronene. J. Am. Chem. Soc. 128, 14337-14340 (2006).

20. Haines-Butterick, L. et al. Controlling hydrogelation kinetics by peptide design for three-dimensional encapsulation and injectable delivery of cells. Proc. Natl Acad. Sci. USA 104, 7791-7796 (2007).

21. Zhang, S. Fabrication of novel materials through molecular self-assembly. Nature Biotechnol. 21, 1171-1178 (2003).

22. Banwell, E. F. et al. Rational design and application of responsive alpha-helical peptide hydrogels. Nature Mater. 8, 596-600 (2009).

23. Adler-Abramovich, L. et al. Self-assembled arrays of peptide nanotubes by vapour deposition. Nature Nanotech. 4, 849-854 (2009).

24. Smith, A. M. et al. Fmoc-diphenylalanine self assembles to a hydrogel via a novel architecture based on pi-pi interlocked beta-sheets. Adv. Mater. 20, 37-41 (2008)

25. Zhang, Y., Gu, H., Yang, Z. \& Xu, B. Supramolecular hydrogels respond to ligand-receptor interaction. J. Am. Chem. Soc. 125, 13680-13681 (2003).

26. Keller, D. \& Bustamante, C. Theory of the interaction of light with large inhomogeneous molecular aggregates. II. psi-type circular dichroism. J. Chem. Phys. 84, 2972-2980 (1986)
27. Martin, J. E. \& Wilcoxon, J. P. Critical dynamics of the sol-gel transition. Phys. Rev. Lett. 61, 373-376 (1988).

28. Fang, L., Brown, W. \& Konak, C. Dynamic light scattering study of the sol-gel transition. Macromolecules 24, 6839-6842 (1991).

29. Weijers, M., Visschers, R. W. \& Nicolai, T. Light scattering study of heat-induced aggregation and gelation of ovalbumin. Macromolecules 35, 4753-4762 (2002).

30. Yamamoto, Y. et al. Photoconductive coaxial nanotubes of molecularly connected electron donor and acceptor Layers. Science 314, 1761-1764 (2006)

31. Schenning, A. P. H. J. \& Meijer, E. W. Supramolecular electronics; nanowires from self-assembled $\pi$-conjugated systems. Chem. Commun. 26, 3245-3258 (2005).

32. $\mathrm{Xu}, \mathrm{H}$. et al. An investigation of the conductivity of peptide nanotube networks prepared by enzyme-triggered self-assembly. Nanoscale 2, 960-966 (2010).

33. Ashkenasy, N., Seth Horne, W. \& Ghadiri, M. R. Design of self-assembling peptide nanotubes with delocalized electronic states. Small 2, 99-102 (2006).

\section{Acknowledgements}

The authors acknowledge the Engineering and Physical Sciences Research Council (EPSRC), Human Frontiers Science Programme (HFSP) and the Leverhulme Trust (UK) for funding. The authors also thank L. Birchall and P.F. Caponi for assistance with graphics.

\section{Author contributions}

A.R.H., S.R., J.S., S.S. and R.V.U. conceived and designed the experiments. A.R.H., S.R., M.A., A.K.D., N.H., N.J. and J.B. performed the experiments. A.R.H., S.R., P.M., J.S., S.S. and R.V.U. analysed the data. S.M., J.H.v.E. and N.T.H. contributed materials/analysis tools. A.R.H., S.R., S.S. and R.V.U. co-wrote the paper.

\section{Additional information}

The authors declare no competing financial interests. Supplementary information and chemical compound information accompany this paper at www.nature.com/ naturechemistry. Reprints and permission information is available online at http://npg.nature. $\mathrm{com} /$ reprintsandpermissions/. Correspondence and requests for materials should be addressed to R.V.U. 